Chang, S. L. (1958). J. gen. Microbiol. 18, 579-585

\title{
Cytological and Ecological Observations on the Flagellate Transformation of Naegleria gruberi
}

\author{
By S. L. CHANG \\ Robert A. Taft Sanitary Engineering Center, Bureau of State Services, \\ Public Health Service, U.S. Department of Health, Education, \\ and Welfare, Cincinnati, Ohio, U.S.A.
}

SUMMARY: Phase-contrast microscopic examination of Naegleria gruberi (Schardinger) during flagellate transformation and reversion revealed the following cytological features. Flagella were formed by filamentous extension from endoplasmic protrusion. One, two or three pairs of flagella were formed from a single protrusion. Occasionally a second protrusion gave rise to the 3rd pair of flagella. A chromatin body of cytoplasmic origin was always present at the base of the protrusion or of the flagella, and is believed to be the parabasal body. Occasionally one chromatin body was seen at the base of each pair of flagella. Alternating bands of light and dark areas were seen in the flagella soon after they were formed and persisted throughout the flagellate stage. Reversion from flagellate to amoeba stage was accomplished by absorption of the flagella, the shedding of one or more flagella and the absorption of the rest, or by casting-off a small part of the body to which the flagella were attached. The following effects of environmental factors on flagellate transformation were observed. As many as $35 \%$ of the trophozoites underwent flagellate transformation in water when they were in metacystic stage or were harvested from plate cultures up to $12 \mathrm{hr}$. of incubation at $25-27^{\circ}$; as the cultural age advanced, the $\%$ amoebae undergoing transformation became progressively lower. Prolonged cultivation in a liquid medium at $25-27^{\circ}$, or on an agar medium at $37^{\circ}$, resulted in temporary loss of ability to form flagellates. The freezing of trophozoites up to $\mathbf{4 0} \mathrm{min}$. and of cysts up to 1 week at $-25^{\circ}$ to $-30^{\circ}$ stimulated flagellate transformation. An increase in water temperature from $25-27^{\circ}$ to $37^{\circ}$ or decrease to 8-10 ${ }^{\circ}$ suppressed the transformation, but after the flagellates were formed, the former temperature change shortened while the latter lengthened duration of the flagellate stage. Freezing of the amoebae in the flagellate stage at $-25^{\circ}$ to $-30^{\circ}$ exerted no adverse effect on their return to amoeboid form when the freezing time did not exceed $30 \mathrm{~min}$. Cultures developed from single organisms isolated in the flagellate stage yielded amoebae showing flagellate transformation characteristics similar to those yielded by ordinary cultures.

Detailed study of the transformation of Naegleria gruberi (Schardinger) from amoebae to flagellates and vice versa is yet to be made. With the strain of $N$. gruberi maintained in rich permanent cultures as described in the preceding paper (Chang, 1958), observations were made on the cytology of flagellate transformation and reversion to amoebae with a phase-contrast microscope, and also of the effects of various environmental factors on this morphological transformation.

\section{METHODS}

Pure mixed cultures of Naegleria gruberi and Proteus mirabilis were maintained at room temperature on buffered sucrose nitrate agar (Chang, 1958). For this study, the mucoid growth from a young plate culture was suspended in a small 
amount of distilled water and examined with a phase-contrast microscope. To immobilize the flagellates for photomicrography, both cold and heat proved unsatisfactory, but a concentration of $c .5 \mu \mathrm{g} . \mathrm{AgNO}_{3} / \mathrm{ml}$. in the suspension successfully immobilized the flagellates with fewer protoplasmic changes than other chemicals. In order to observe the cytological characteristics of the flagellate forms that were obliterated by the $\mathrm{AgNO}_{3}$ treatment, photomicrographs were taken of those about to undergo reversion and moving slowly enough to permit photomicrography. Because of the limited amount of light transmitted by the phase-contrast microscope, exposure times of a few seconds were required and some blurring was unavoidable. To develop pure-line cultures of $N$. gruberi from single organisms in the flagellate stage, flagellates induced in sterile distilled water were fished out singly with micropipettes and placed on agar plates pre-seeded with $P$. mirabilis. All inoculated plates were thoroughly examined under a low power, and only those with a

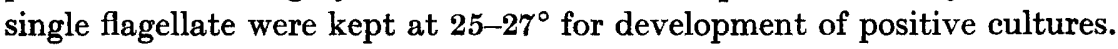

\section{RESULTS}

\section{Cytology of the flagellate tránsformation}

At the start of the flagellate transformation, the trophozoites formed pseudopodia and retained motility, but at the same time they protruded an endoplasmic mass. After the protrusion reached a certain size, it constricted at the base. The apparent homogeneous mass formed a pair of flagella by extending two long filamentous processes, one after the other (Pl. 1, fig. 1). In the formation of two or three pairs of flagella, corresponding processes were extended from the protruded mass. Occasionally a second protrusion formed near the first one, for the formation of the 3rd pair of flagella. Formation of more than three pairs of flagella was not observed. The report of Wenyon (1926) that one, two, or three pairs of flagella were formed in individuals having the corresponding number of nuclei was not confirmed. In all cases of flagellate transformation observed, the trophozoites were exclusively uninucleate.

While the flagella were being formed, a chromatin body of cytoplasmic origin was always present at the base of the protrusion. After the flagella were formed, this chromatin body was located at the base of the flagella and is believed to be the basal body. When more than one pair of flagella were formed, a basal body might be present at the base of each pair (Pl. 1, fig. 2). The statements of Alexieff (1912) and of Wilson (1916) that one blepharoplast is present at the base of each flagellum, that it is of karyosome origin and is connected to the flagellum by a rhizoplast, were not confirmed. Soon after the flagella were formed, there appeared to be a short period during which the endoplasm in each flagellum underwent rearrangement to form alternating light and dark bands (Pl. 1, figs. 3, 4), and the ectoplasm formed a thin layer beneath the cytoplasmic membrane. The alternating bands are believed to be of sol and gel and the peripheral arrangement of the ectoplasm to be responsible for the cell wall-like appearance in the flagellate forms (Pl. 2, figs. 5-7). 


\section{Cytology of the flagellate forms}

The alternating light and dark bands in the flagella persisted throughout the flagellate stage. As soon as the flagella exhibited movement, the body became pear-shaped with the flagella located at the pointed end (Pl. 2, figs. 5-7). At this stage, the amoebae lost their identity and looked in every respect like 'flagellates'. Movement of the flagella, whether of one, two or three pairs, was circular and so well co-ordinated that the organisms were propelled forward in a straight course. When a turn was made, the posterior end of the organism tilted toward the direction of the turn.

Although the appearance of the alternating bands was abolished by the use of $\mathrm{AgNO}_{3}$, the flagella, the basal body and the nucleus were easily recognizable. The basal body at the base of the flagella was very close to the cytoplasmic membrane (Pl. 1, fig. 2; Pl. 2, fig. 5). The nucleus might be located in any part of the body, although most frequently it was in the anterior one-third (Pl. 2, figs. 5-7). The large contractile vacuole situated near the posterior end, as described by Singh (1952), was frequently absent. When present, it might be located in any part of the posterior half. When two pairs of flagella developed, they were arranged very close to each other (Pl. 2, fig. 7). In some flagellate forms there appeared to be a cytostome-like structure, as suggested by Wenyon (1926), along one side of the anterior end (Pl. 2, fig. 6). Repeated examination of a large number of these forms revealed that this groove-like appearance was caused by the slight twisting of the anterior end as the organisms were propelled forward in a rotary motion.

\section{Abnormal flagellate forms}

During the flagellate transformation, there were significant numbers of trophozoites which showed such abnormal development that they failed to complete the transformation. These abnormal forms were generally not recognized in ordinary microscopic examination. In some cases, elongation of the endoplasmic protrusion resulted in the formation of a long filamentous process with a substantial piece of endoplasm remaining at the end, which might or might not show signs of bifurcation. Some trophozoites developed structures like tree-branches from the protruded mass; some formed numerous short processes without flagella formation. Still others formed multiple protrusions, some of which extruded long processes while others became detached. The first three types of abnormal forms usually reverted to the amoeba stage; the last types often disintegrated.

\section{Cytology of reversion}

Because of the non-feeding nature of the flagellate forms, the ceaseless motion apparently results in a rapid exhaustion of stored food. It is believed that return to the amoeba forms is stimulated as this stored food is consumed. Generally speaking, after $2-3 \mathrm{hr}$. of continuous movement, the flagellates frequently showed a change of body shape. Since the ectoplasm is distributed in a thin layer under the cytoplasmic membrane, bulges contain mostly endo- 
plasm. As a result of changes in body shape, forward movement tended to be irregular rather than straight. Soon the organisms became more and more amoeboid in shape, with movement becoming slower and more irregular. Eventually, the shapes became so altered that the propelling action of the flagella resulted in a rotary motion without much change in position. Finally movement stopped, although the flagella might continue to show some weak circular motion for a few seconds. As soon as they completely stopped movement, the alternating light and dark bands disappeared, giving further evidence that they were intimately associated with movement.

There appears to be at least three ways by which flagellate forms revert to amoeboid forms. In the majority of cases, reversion was accomplished by a continuous shortening of the flagella, resulting in the formation of beaded processes with progressively fewer but larger beads until the whole process was absorbed by the body (Pl. 2, fig. 8). Rafalko (1947) considered this the only means of amoeba reversion. Less frequently, reversion was achieved by dropping parts of one or more flagella and absorbing the rest. In a very few individuals, the return was completed by the formation of a constriction near the base of a flagellum, which eventually separated the flagellum and a small piece of endoplasm from the rest of the organism (Pl. 2, fig. 9). Following the first two types of reversion, the amoebae invariably encysted. The fate of the amoebae after the last type of reversion is unknown.

\section{Effects of environmental factors on flagellate transformation}

Cultural age. By using newly excysted amoebae and trophozoites harvested from plate cultures of different incubation periods, the influence of the cultural age on the flagellate transformation was studied; the results obtained are shown in Table 1.

Table 1. Effect of incubation time of plate cultures of Naegleria gruberi on the flagellate transformation in water

\begin{tabular}{|c|c|}
\hline $\begin{array}{c}\text { Cultural age } \\
(\text { hr.) }\end{array}$ & $\begin{array}{c}\text { Flagellate } \\
\text { transformation }(\%)\end{array}$ \\
\hline Metacystic amoeba & 30-35 \\
\hline $4-8$ & 25-35 \\
\hline $12-14$ & 20-30 \\
\hline 24-32 & $10-15$ \\
\hline $48-56$ & $5-10$ \\
\hline $60-72$ & $1-5$ \\
\hline $80-96$ & $<1$ \\
\hline
\end{tabular}

Cultivation in a fluid medium. Trophozoites harvested from cultures in buffered sucrose tryptose broth (Chang, 1958) after different incubation periods and concentrated by low-speed centrifugation, were suspended in distilled water to induce flagellate transformation. The results in repeated experiments showed that the amoebae lost ability to form flagellates after $48 \mathrm{hr}$. or longer in the broth. By transferring amoebae from the liquid medium to the agar medium, flagellate transformation was regained after two or more subcultures; but the longer the organism was maintained in broth cultures, 
the more subcultures on solid medium were required to regain the ability to transform. For example, five to six plate subcultures (6-8 weeks) were required to retain this ability after the amoeba had remained in broth cultures for 4-5 weeks.

Cultivation on an agar medium at $37^{\circ}$. Repeated attempts were made to induce flagellate transformation of newly excysted amoebae and young trophozoites harvested from plate cultures maintained at $37^{\circ}$. Results indicated that the amoeba lost its flagellate-forming ability after two or more subcultures ( 2 weeks or longer) at $37^{\circ}$. This phenomenon was temporary and the ability was regained by the amoeba after several transfers at $25-27^{\circ}$. It was not ascertained whether or not longer cultivation at $37^{\circ}$ would result in permanent loss of this ability.

Freezing at $-25^{\circ}$ to $-30^{\circ}$. Water suspensions of trophozoites of various cultural ages were frozen at $-25^{\circ}$ to $-30^{\circ}$ shortly after preparation to avoid flagellate transformation. Samples were removed one at a time, thawed, and examined for flagellate forms. The results revealed that freezing for 10-40 $\mathrm{min}$. increased flagellate transformation by about $15 \%$ over that observed with unfrozen amoebae of the same cultural age; freezing for more than $40 \mathrm{~min}$. resulted in death.

The next step was to determine how freezing would effect survival of the flagellates and reversion to the amoeba forms. Water suspensions of trophozoites of $c .12 \mathrm{hr}$. cultural age were allowed to stand until a maximum number of flagellates was formed. These were then frozen, thawed, and examined for motility and reversion as described above. The results indicated that freezing at $-25^{\circ}$ to $-30^{\circ}$ up to $40 \mathrm{~min}$. exerted no adverse effect on these activities. Like the trophozoites, the flagellate forms were killed when the freezing time was extended over 40 min.

It is interesting to note that while the flagellate transformation was never observed on agar plates in routine cultures, it was repeatedly noted during the first few hours of incubation on plates seeded with cysts which had previously been frozen at -25 to $-30^{\circ}$ from a few hours to about 1 week. Frequent examinations during the early hours of incubation revealed that many of the newly excysted amoebae underwent flagellate transformation soon after they emerged. The number of newly excysted amoebae undergoing transformation became progressively less as freezing time of the cysts was lengthened; none formed flagellates after $>3$ weeks of freezing.

\section{Effect of temperature on flagellate transformation and duration of the flagellate stage}

A water suspension of young trophozoites was dispensed in five Petri dishes and handled in the following manner: one was placed in a refrigerator at 8-10 ${ }^{\circ}$, one in an incubator at $37^{\circ}$, and three were left at $25-27^{\circ}$. All dishes were examined at hourly intervals for flagellates. As soon as the number of flagellates reached the peak in the dishes left at $25-27^{\circ}$ one dish was moved to the refrigerator, one to the $37^{\circ}$ incubator, and the third remained at $25-27^{\circ}$. Examination of all dishes continued until the flagellates returned to amoebae. 
In three replicate experiments it was found that storage of the trophozoites at $8-10^{\circ}$ or at $37^{\circ}$ suppressed the flagellate transformation, and that while most of the transformed organisms returned to amoebae within $6 \mathrm{hr}$. at $25-27^{\circ}$, the flagellate stage was lengthened to $8-10 \mathrm{hr}$. at $8-10^{\circ}$ and was shortened to c. $2 \mathrm{hr}$. at $37^{\circ}$. Flagellate motility at $8-10^{\circ}$ was noticeably slower and at $37^{\circ}$ somewhat faster than at $25-27^{\circ}$.

\section{Flagellate transformation of trophozoites from pure-line cultures developed from single flagellates}

Of a total of ten isolations made in the flagellate stage, five developed positive amoeba cultures. These pure-line cultures resembled others in cultural appearance and in growth and encystation characteristics. By suspension in water they exhibited flagellate transformation activity identical with that of ordinary cultures.

\section{DISCUSSION}

The development of flagella is the most fascinating phenomenon in the whole process of flagellate transformation. Some internal rearrangement of the endoplasm must take place before the formation of the protrusion in order to provide homogeneous material for the formation of the flagella, and of a chromatin body of cytoplasmic origin for the formation of the basal body or bodies. The alternating light and dark bands in the flagella appear to be bands of sol and gel and are believed to be responsible for the motility of the flagella. A central contractile fibril has been demonstrated in the flagella of some large flagellates; however, the presence of such a fibril has not been demonstrated in smaller forms such as Trichomonas and Chilomastix spp. It would be interesting to determine whether the mechanism of flagella motility in these forms is similar to that of the transformed flagellates of Naegleria gruberi. In most true flagellates, the anterior end is the more blunt, enabling the organisms to swim with the least amount of hydraulic resistance. In the transformed flagellates of $N$. gruberi, the anterior end is more pointed, due to the lack of a sufficiently rigid wall around the organisms to maintain the typical 'fish' shape when the hydraulic pressure exerted at the anterior end of the moving organisms is greater than at the other points.

The observations obtained on the effects of various environmental factors on flagellate transformation of Naegleria gruberi justify the following general statements: (1) flagellate transformation is an activity of this flagellate amoeba, transmissible through its offspring on a fixed percentage basis; (2) the potentiality of flagellate transformation is at its peak in newly excysted amoebae and in very young trophozoites, decreasing steadily as the cultural age advances; trophozoites of very old cultures lose most of this potentiality; (3) the mechanism of flagellate transformation is probably triggered by hydration of a certain part of the cytoplasm of the amoeba and occurs when newly excysted amoebae or plate-grown trophozoites are suspended in water; this hydration effect, however, may be obliterated by growing the amoeba in a fluid medium or by prolonged culitvation at $37^{\circ} ;(4)$ freezing of trophozoites 
Journal of General Microbiology, Vol. 18, No. 3
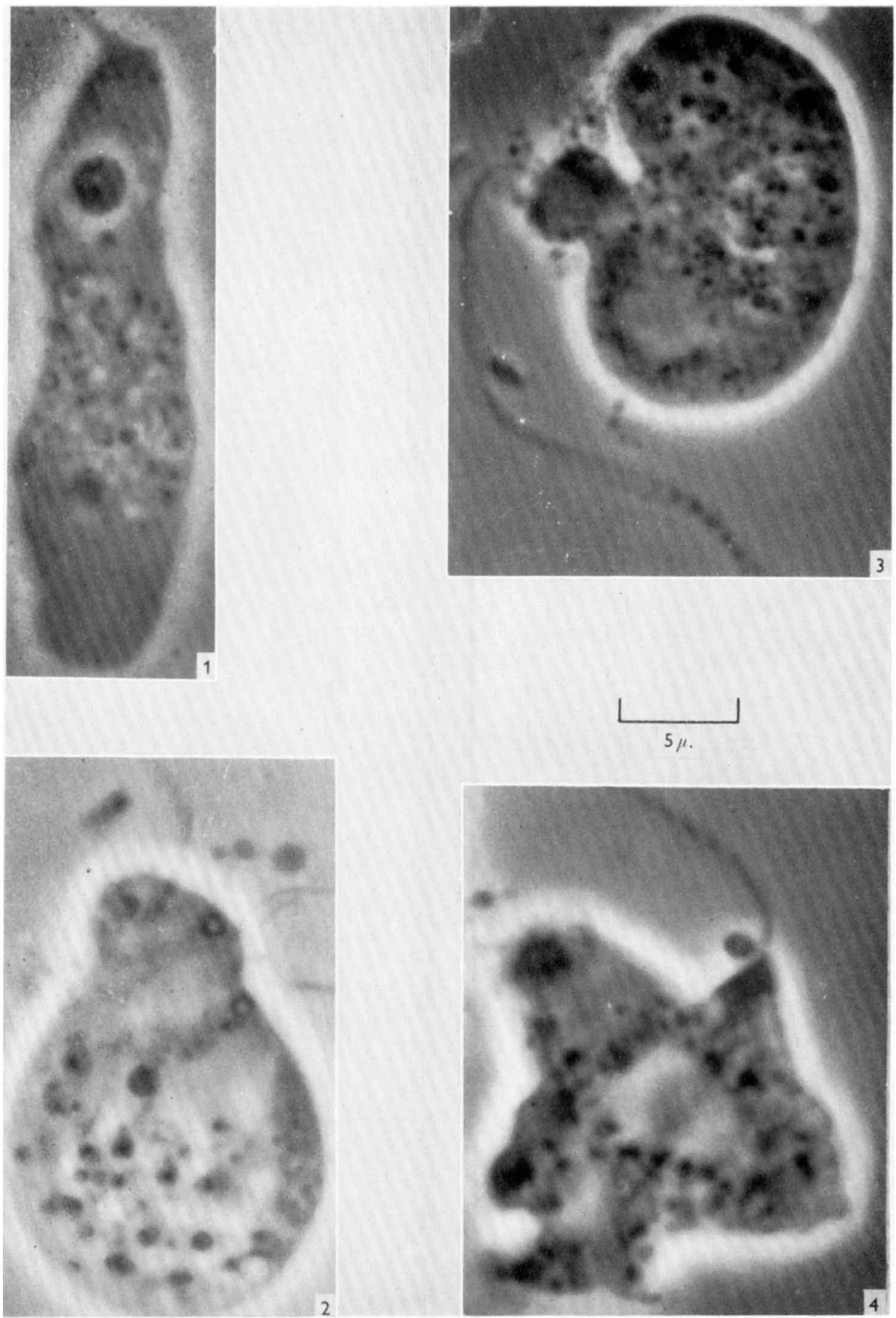

S. L. Chang-Transformation of Nabgheria gruberi. Plate 1 
Journal of General Microbiology, Vol. 18, No. 3
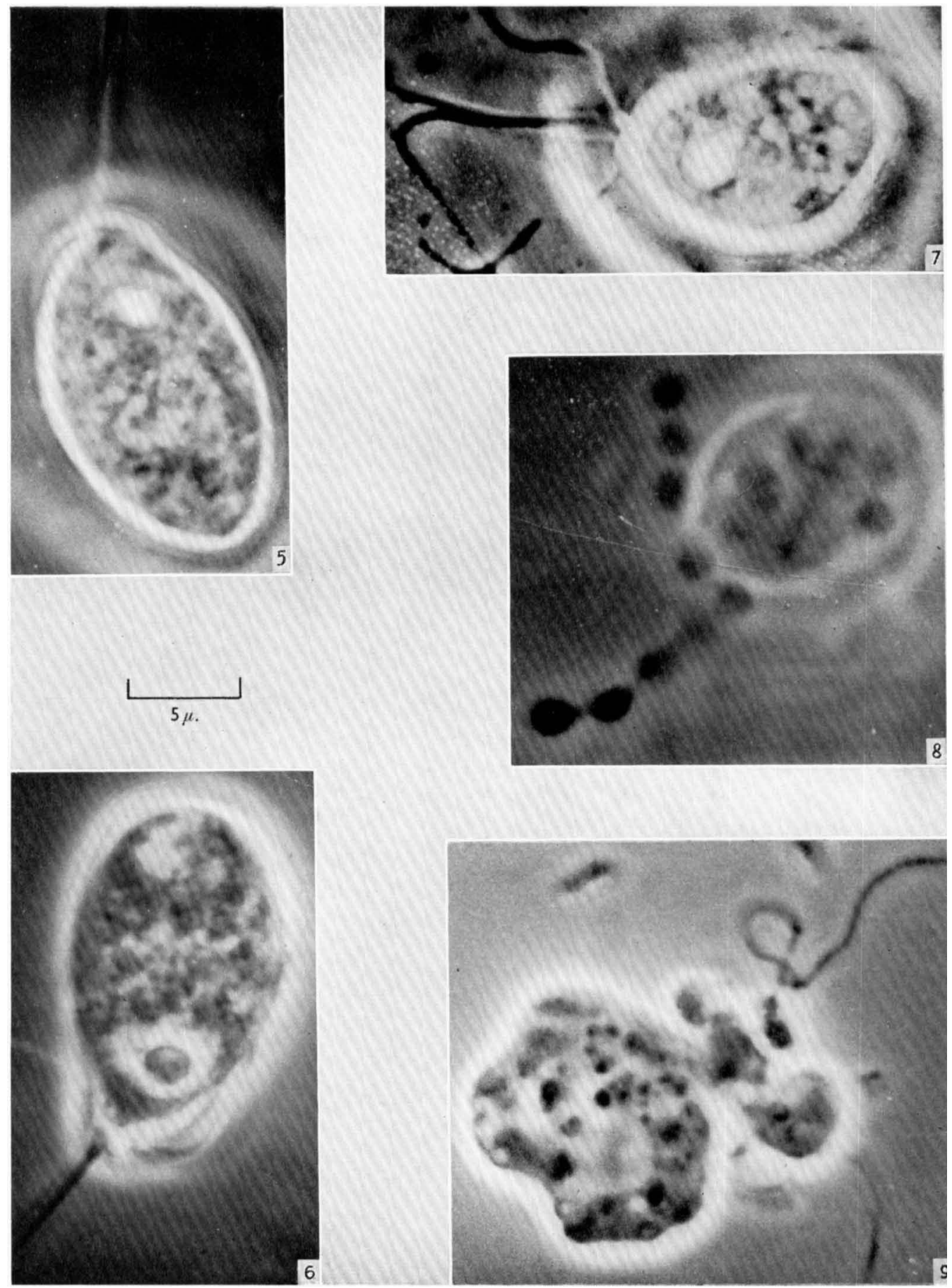

S. L. Chang-Transformation of Naegleria gruberi. Plate 2 
within the survival period and of cysts up to about 1 week increased the potentiality of flagellate transformation, probably due to dehydration during freezing.

Observations on the prolonging effect of low temperatures and on the shortening effect of high temperatures of the flagellate stage, support the statement that flagellates are non-feeding forms and their ceaseless motion results in a rapid exhaustion of stored food, stimulating return to the amoeboid form. The higher the temperature, the more rapid is the movement and the more rapid is the exhaustion of the stored food.

\section{REFERENCES}

Alexiefr, A. (1912). Sur les caractères cytologiques et la systématique des amibes du groupe limax (Naegleria nov. gen et Hartmannia nov. gen) et des amibes parasites des vertébrés (Proctamoeba nov. gen). Bull. Soc. zool. Fr. 37, 55.

Chang, S. L. (1958). Cultural, cytological and ecological observations on the amoeba stage of Naegleria gruberi. J. gen. Microbiol. 18, 565.

Rafalko, J. S. (1947). Cytological observations on the amoeba-flagellate, Naegleria gruberi (Protozoa). J. Morph. 81, 1.

SiNGH, B. N. (1952). Nuclear division in nine species of small free-living amoebae and its bearing on the classification of the order Amoebida. Phil. Trans. B, 236, 405.

Wenyon, C. M. (1926). Protozoology. London: Baillière, Tindall and Cox.

Wilson, C. W. (1916). On the life history of a soil amoeba. Univ. Calif. Publ. Zool. 16, 241.

\section{EXPLANATION OF PLATES}

Plate 1

Phase-contrast micrographs of flagellate forms of $N$. gruberi.

Fig. 1. A trophozoite during the early stage of flagellate transformation.

Fig. 2. A flagellate form showing basal body at the base of each pair of flagella. Notice the change to amoeboid shape.

Figs. 3, 4. Late flagellate forms showing the alternating light and dark bands in flagella. Notice the change to amoeboid shape.

\section{Plate 2}

Figs. 5-7. Flagellate forms immobilized by $\mathrm{AgNO}_{3}$. Notice the basal body in fig. 5, cytostome-like appearance in fig. 6, two pairs of flagella in fig. 7, and anteriorly located nucleus in all.

Figs. 8, 9. Flagellate forms reverting to amoebae. Notice the beaded appearance of flagella in fig. 8 and the separation of a small piece of cytoplasm and flagella from the body in fig. $\theta$.

(Received 30 September 1957) 